\title{
Balance of cardiac and systemic hepcidin and its role in heart physiology and pathology
}

Driton Vela

Hepcidin is the main regulator of iron metabolism in tissues. Its serum levels are mostly correlated with the levels of hepcidin expression from the liver, but local hepcidin can be important for the physiology of other organs as well. There is an increasing evidence that this is the case with cardiac hepcidin. This has been confirmed by studies with models of ischemic heart disease and other heart pathologies. In this review the discussion dissects the role of cardiac hepcidin in cellular homeostasis. This review is complemented with examination of the role of systemic hepcidin in heart disease and its use as a biochemical marker. The relationship between systemic vs local hepcidin in the heart is important because it can help us understand how the fine balance between the actions of two hepcidins affects heart function. Manipulating the axis systemic/cardiac hepcidin could serve as a new therapeutic strategy in heart diseases.

Laboratory Investigation (2018) 98, 315-326; doi:10.1038/labinvest.2017.111; published online 23 October 2017

\section{IRON, A CRUCIAL CELLULAR MICRONUTRIENT: ROLE AND TRANSPORT}

Iron is an essential nutrient for cell metabolism. It is involved in DNA synthesis, ATP production, and oxygen transport. ${ }^{1,2}$ However, iron can be toxic for cells when they get overloaded by it. ${ }^{1}$ This happens because of the ability of excess iron to produce reactive oxygen species (ROS). ${ }^{1}$ On the other hand, iron is important for survival of pathogenic microbes. ${ }^{1}$ These observations mean that in order for our cells to get the most benefits out of iron, there needs to exist a fine-tuning of the machinery of proteins that can control iron reactivity and availability. Most of the iron in our body comes from dietary sources. However, iron ingested with food is in trivalent form, which is not suitable for cellular reactions. ${ }^{1,3}$ Therefore, enterocytes first reduce the trivalent form of iron into a divalent form through enzymes called reductases. ${ }^{1,3}$ After reduction divalent iron is transported inside enterocytes through an iron transporter named divalent metal transporter 1. ${ }^{1}$ Once inside cells, iron is linked with chaperones like poly(rC)-binding proteins. ${ }^{3}$ PSBPs act as intracellular iron transporters delivering iron to ferritin, but also to proteins that use iron as a cofactor. ${ }^{3}$ Ferritin serves as a cellular iron depot capable of storing high amount of iron atoms. ${ }^{3}$

Iron export out of cells is attributed to ferroportin (FPN), but the mechanisms that involve the delivery of iron from intracellular environment to FPN are not well understood. ${ }^{3}$ Role of FPN as a cellular iron exporter is linked with the activity of ferroxidases that turn iron into a trivalent form. ${ }^{3}$ Only in this chemical form iron can bind to its transporter in the blood, called transferrin., ${ }^{1,3}$ Transferrin circulates in the blood and finally binds to its receptor, transferrin receptor 1 (TFR1), which delivers iron inside cells such as erythroblasts, hepatocytes, and macrophages. ${ }^{1,3}$ The intracellular iron homeostasis is regulated by iron regulatory proteins (IRPs). ${ }^{4}$ Under conditions characterized by low intracellular iron, IRPs bind to iron-responsive elements (IREs) of TFR mRNA and FPN mRNA. ${ }^{4}$ This action of IRPs will cause increased expression of TFR1 and decreased expression of FPN. ${ }^{4}$ The aim of these changes is to increase iron supply through TFR1 and reduce iron export through downregulation of FPN. When intracellular iron is abundant, the active center of IRPs is blocked and cannot bind to IREs. ${ }^{4}$

\section{HEPCIDIN, THE PRIME CONTROLLER OF IRON METABOLISM}

Our bodies control serum iron and cellular iron levels through different proteins, of which, a small peptide named hepcidin has been established as the major regulator of iron metabolism. It exists in three main isoforms: hepcidin-25, -22 , and -20 , of which hepcidin-25 is considered the main physiological actor in iron homeostasis. ${ }^{5}$ It is produced by hepatocytes mainly in response to iron-mediated pathways. ${ }^{6}$ Iron-mediated pathways induce signaling through bone morphogenetic protein 6 (BMP6), HFE protein, and 


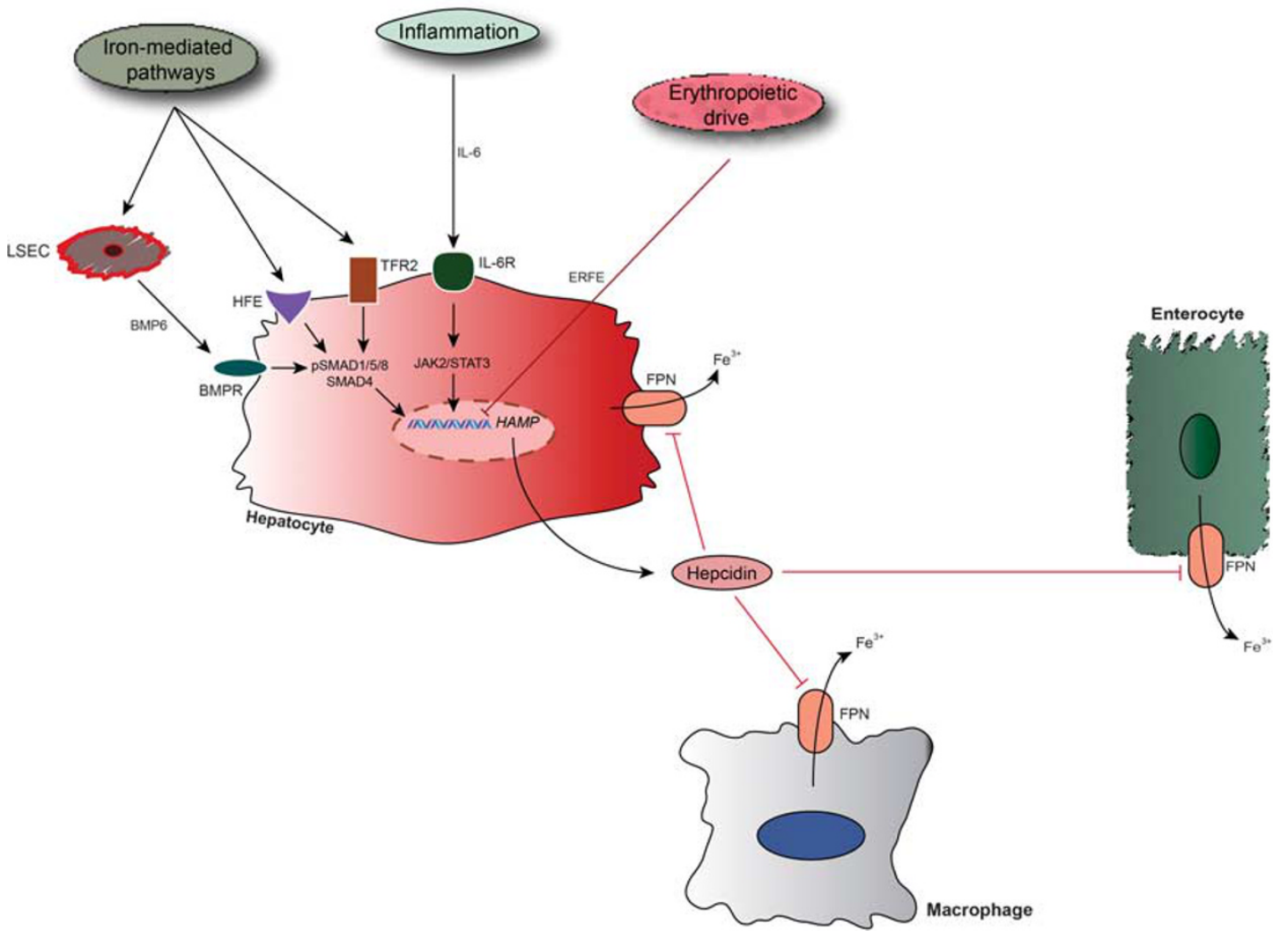

Figure 1 Regulation and action of liver (systemic) hepcidin. Regulators of hepcidin expression in hepatocytes are iron-mediated pathways, inflammatory signals, and erythropoietic drive. Iron-mediated pathways act through BMP6, TFR2, and HFE. BMP6 is produced by non-parenchymal liver cells (like LSEC). BMP6 binds with BMPR that activates the SMAD pathway. Phosphorylated SMAD molecules induce HAMP expression. Inflammation is another major regulator of hepcidin expression that acts by activating the JAK2/STAT3 pathway. Erythropoietic drive suppresses hepcidin expression through newly discovered ERFE. Hepcidin induces FPN degradation in effector cells, which causes sequestration of iron in cells. This is accompanied with reduction in iron plasma levels. BMP6, bone morphogenetic protein 6; BMPR, BMP receptor; ERFE, erythroferrone; FPN, ferroportin; HAMP, hepcidin antimicrobial peptide; HFE, hemochromatosis protein; JAK2/STAT3, janus kinase 2/signal transducer and activator of transcription 3; LSEC, liver sinusoidal endothelial cells; SMAD, S-mothers against decapentaplegic; TFR2, transferrin receptor 2.

transferrin receptor 2 (TFR2). ${ }^{7,8}$ BMP6 is a molecule produced by nonparenchymal liver cells in response to iron load (Figure 1). ${ }^{9}$ It acts in a paracrine way by binding to BMP receptor (BMPR) in hepatocytes. ${ }^{10}$ BMPR actions are modulated by its co-receptor, hemojuvelin (HJV). ${ }^{11}$ This interaction activates the intracellular s-mothers against decapentaplegic (SMAD) pathway that will eventually upregulate hepcidin expression. ${ }^{12}$ HFE and TFR2 are also mediators of iron-mediated signaling in hepatocytes by acting as membrane receptors that react to transferrin and probably other molecules. It is still not clear how do HFE and TFR2 cause upregulation of hepcidin, but TFR2 seems to be more potent in this respect compared with HFE. ${ }^{8}$ This is also the reason why the clinical course of classical hemochromatosis (HH) caused by HFE mutations is more benign compared with $\mathrm{HH}$ caused by TFR2 disturbance. ${ }^{13}$ Another important regulator of hepcidin expression is inflammatory signals. They act through cytokines like interleukin-6 (IL-6), which activates IL-6 receptor (IL-6R). ${ }^{14,15}$ Activated IL-6R upregulates the janus kinase 2/signal transducer and activator of transcription 3 (JAK2/STAT3) pathway. ${ }^{15}$ STAT3 has a binding site in the hepcidin antimicrobial peptide (HAMP) promoter that causes increased expression of hepcidin. ${ }^{15}$ Inflammatory signals and iron-mediated pathways are connected through actions of activin $\mathrm{B}$, but the importance of this connection is debatable. ${ }^{16,17}$ Apart from positive regulators of hepcidin, there are a number of negative regulators that suppress hepcidin expression. Signaling from erythrocyte precursors (through newly discovered erythroferrone (ERFE)) causes downregulation of hepcidin expression, which serves as a mechanism for increased iron delivery into cells that need iron for the assembly of hemoglobin molecules. ${ }^{18}$ Other suppressors of hepcidin expression include hypoxia, vitamin $\mathrm{D}$, and estrogen. ${ }^{19-21}$

The main mode of action of hepcidin is realized through FPN. $^{22,23}$ Hepcidin binds with FPN and causes FPN degradation inside lysosomes of the cells. ${ }^{23}$ In this way hepcidin lowers the activity of the main exporter of iron from cells. This means iron cannot get out of cells and into the blood. The most significant action of hepcidin in sequestering 
iron into cells is observed in macrophages, enterocytes, and hepatocytes. ${ }^{22}$ These cells are responsible for maintaining high turnover of iron; therefore, the action of hepcidin through FPN is crucial in securing strict levels of iron availability for cells. This role is even more important when one considers the fact that our body has no known excretory route for iron disposal. ${ }^{24}$

\section{ROLE OF CARDIAC HEPCIDIN IN HEART HOMESTASIS}

Although the role of systemic (liver) hepcidin has been studied extensively, more and more research is shedding light on the importance of local hepcidin in organ homeostasis. There are a number of studies confirming the role of local hepcidin in the lungs, stomach, and prostate. ${ }^{25-27}$

\section{Cardiac HAMP and FPN are More Important Than Liver HAMP for the Regulation of Heart Homeostasis}

Iron metabolism in the heart is not regulated only by the liver (systemic) hepcidin, but by local hepcidin as well. Levels of heart hepcidin are up to 30 times lower than in the liver, but still they seem to have an important role for heart function. ${ }^{28}$ We have to keep in mind that next to the liver, the heart is the organ with the highest expression of hepcidin. ${ }^{29}$ This relatively high expression of hepcidin in the heart raises questions about the role of cardiac hepcidin in heart function.

Similar to the liver, cardiac hepcidin acts through FPN, the main cellular iron exporter (Figure 2). ${ }^{30}$ Specific cardiac FPN knockout in mice causes more severe cardiac dysfunction than systemic hepcidin knockout, although hepcidin knockouts have a higher level of iron load in the heart. ${ }^{31}$ This observation implies that, in cardiac FPN knockouts, iron load is mostly concentrated in cardiomyocytes, whereas in the liver HAMP knockouts, iron load is mostly seen in noncardiomyocytes. ${ }^{31}$ Caution should be taken into account when dealing with cardiac FPN knockouts because contradictory data from different models of mice suggest that only specific cardiac FPN mutations can cause significant heart dysfunction. ${ }^{32}$ It is interesting to notice that in classical $\mathrm{HH}$ cardiac damage is observed later than liver damage, ${ }^{33}$ which is in concert with the specific actions of systemic hepcidin in the heart. ${ }^{31}$ However, in FPN disease organ iron load is mild, and the reason for this is that in systemic FPN dysfunction iron cannot get out from principal cell reservoirs of iron, such as enterocytes and macrophages, thus making cardiac iron load unlikely. ${ }^{34}$ Cardiac FPN controls iron load in cardiomyocytes because iron excess upregulates FPN expression. ${ }^{35}$ Increased activity of cardiac FPN protects cardiomyocytes during iron excess by stimulating iron efflux from cells. ${ }^{31}$

Cardiac hepcidin is emerging as a crucial homeostatic factor for cardiac physiology. Recent research conducted by Lakhal-Littleton et al. in mouse models with specific cardiac hepcidin knockout showed that loss of hepcidin was associated with fatal cardiac dysfunction. ${ }^{28}$ Loss of cardiac hepcidin resulted in very significant increase in left ventricle mass and apoptosis. This dysfunction caused a marked reduction in ejection fraction of rat hearts. ${ }^{28}$ Could it be that cardiac hepcidin mutations are a source of unrecognized form of cardiomyopathy? This intriguing idea should be investigated by future genetic studies. Another important finding from the elegant paper of Lakhal-Littleton et al. has revealed the differences in the liver and heart hepcidin expression during cardiac iron deficiency. It is known that liver hepcidin

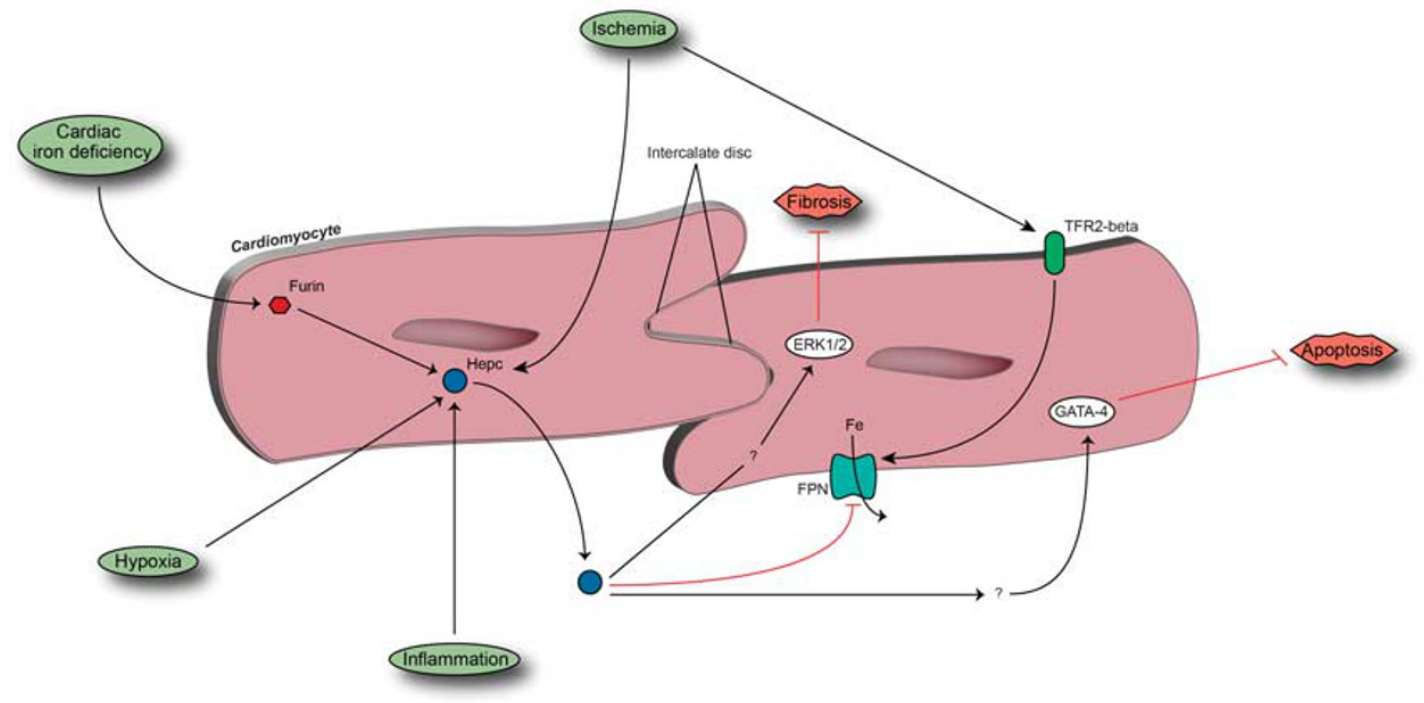

Figure 2 Mode of action of cardiac hepcidin. Cardiac hepcidin is upregulated by ischemia, iron deficiency, hypoxia, and inflammation. Intercalated discs, which function as intercellular links, are the location with the highest concentration of cardiac hepcidin. Cardiac iron deficiency induces hepcidin expression through actions of furin. Cardiac hepcidin similar to the liver acts on cardiac FPN, but can also induce anti-apoptotic (GATA-4) and antifibrotic signals (through ERK1/2 protein mediation). Ischemia is also linked with upregulation of TFR2-beta that counteracts hepcidin actions. ERK, extracellular signal-regulated kinase; FPN, ferroportin; GATA-4, transcription factor; TFR2-beta, transferrin receptor 2 beta. 
Table 1 Overview of the most important findings about cardiac hepcidin actions and regulation

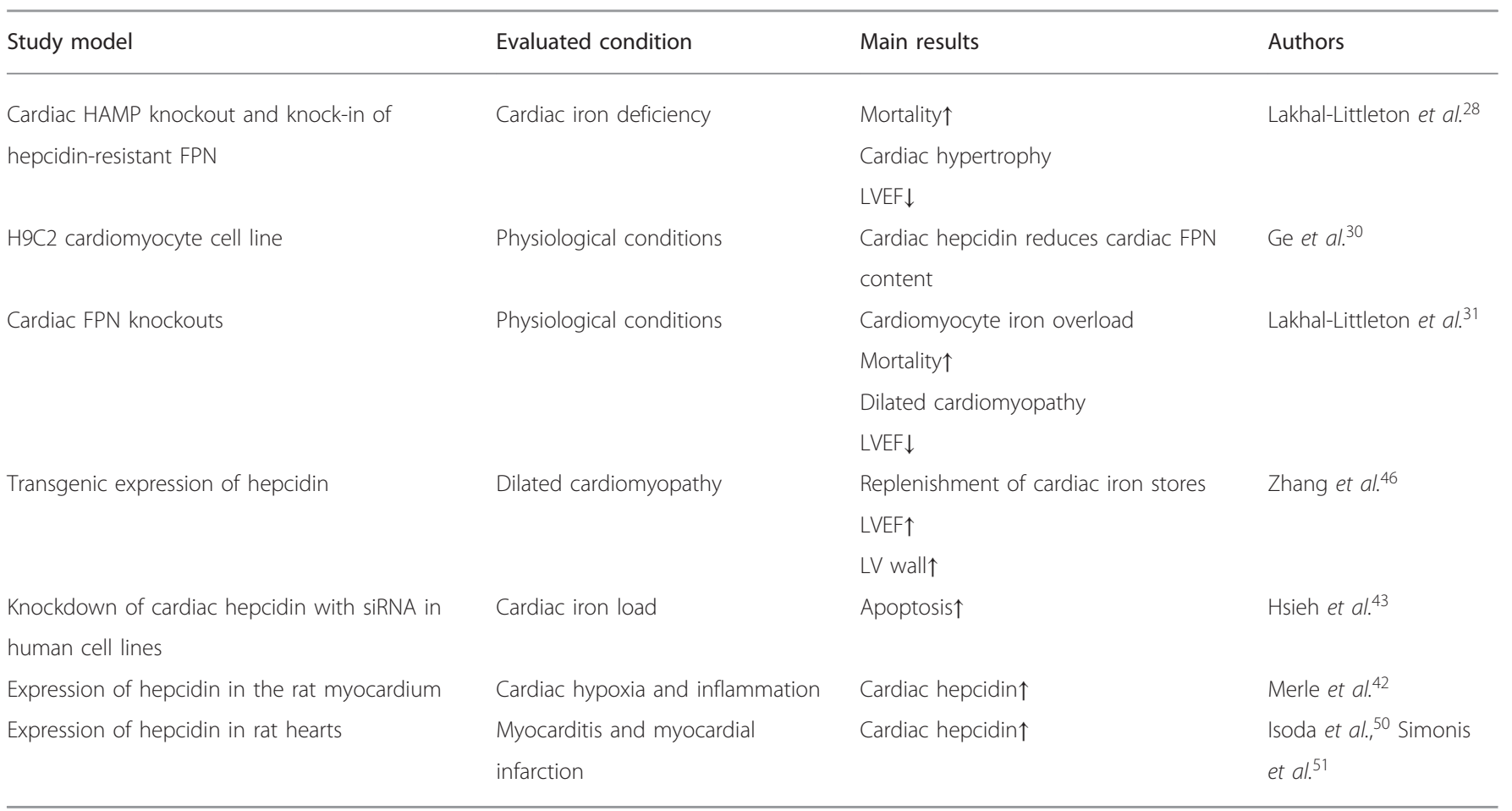

Abbreviations: FPN, ferroportin; HAMP, hepcidin antimicrobial peptide; LV, left ventricle; LVEF, LV ejection fraction; siRNA, small interfering RNA.

is downregulated in iron deficiency. ${ }^{28}$ The downregulation of liver hepcidin serves as a regulatory mechanism that increases iron efflux from enterocytes and macrophages into plasma. Resultant increase in serum iron secures abundant amounts of this important micronutrient for the needs of different tissues. However, in the heart, increased local hepcidin expression during cardiac iron deficiency, as observed by Lakhal-Littleton et al., could also have a protective role, because during iron deficiency increased heart hepcidin will induce FPN degradation and cause iron sequestration in cardiomyocytes. This action reduces iron leakage from cardiomyocytes and preserves iron depots inside the cells. Indeed, preventive iron treatment is not associated with cardiac dysfunction compared with untreated hepcidin knockout mice. ${ }^{28}$ Low serum hepcidin in anemia in heart failure (HF) and increased cardiac hepcidin expression during induced cardiomyocyte iron deficiency by in vivo and in vitro experiments support the observations that show differential regulation of hepatic and cardiac hepcidin by iron. ${ }^{28,36}$ Although total heart iron content did not change in cardiac hepcidin knockouts, cardiomyocyte iron content was significantly lower compared with controls. Furthermore, the reactive upregulation of the iron importer TFR1 in cardiac hepcidin and FPN knockouts is consistent with the presence of an iron-deficient state in these cells. Proper iron supply is vital for mitochondrial enzymatic activity during cardiac injury. ${ }^{37,38}$ In addition, data suggest that preserving cardiac iron during iron deficiency protects from $\mathrm{HF}$, which complements previous observations. ${ }^{38}$

It seems that the intracellular mediator of cardiac hepcidin upregulation during iron deficiency involves furin. ${ }^{28}$ Furin is a protein that cleaves the immature form of hepcidin in its active form in the liver. ${ }^{39}$ Its expression has been observed in cardiac cells as well, and it might be that its action in the heart is similar to the liver. ${ }^{40}$

As we can see, studies suggest that the balance between cardiac and systemic hepcidin is crucial in fine-tuning of cardiac iron transport. Whereas global organ iron supply is mostly controlled by systemic hepcidin, cardiac hepcidin exerts its role by influencing iron efflux in cardiomyocytes (through cardiac FPN). The local scope of cardiac hepcidin and FPN is enforced by results from Lakhal-Littleton et al., ${ }^{28}$ where cardiac hepcidin and FPN-specific knockouts do not show significant changes compared with controls in relation to serum hepcidin levels and global serum iron parameters.

In certain cardiac pathologies characterized with inflammatory infiltrate, local hepcidin can be overexpressed, but the source of this hepcidin is not cardiomyocytes. In Friedreich's ataxia (FA) heart dysfunction is accompanied with chronic myocarditis. ${ }^{41}$ Inflammatory infiltrate in FA shows higher levels of hepcidin expression compared with cardiomyocytes. ${ }^{41}$ This observation should be taken into account when measuring hepcidin expression in the heart, especially in pathologies characterized with excessive inflammatory infiltrate. This is probably the reason why in FA local iron load in cardiomyocytes 
is not in relation to the amount of cardiac damage, which is in contrast with $\mathrm{HH}$, where damage to the heart is not characterized with infiltration by inflammatory cells, but by global excessive iron storage in cardiomyocytes. ${ }^{42}$

\section{Role of Cardiac Hepcidin Extends Beyond Iron Regulation?}

Studies have shown that hepcidin in cardiomyocytes tends to accumulate near intercalate discs, especially in hypoxic conditions, which is interesting, as these intercellular contacts in the heart are crucial for propagating electrical impulses without resistance. ${ }^{43}$ How this relates local hepcidin disturbance with cardiac arrhythmia remains to be seen. Another interesting finding has been observed by silencing hepcidin expression using small interfering RNA. Silencing hepcidin promotes apoptosis in cardiomyocytes, which suggests a protective role of hepcidin beyond iron regulation. ${ }^{44}$ This is in terms with the observation that GATA-4, the transcription factor known for mediating cardiac hypertrophy and apoptosis, has a binding site in the hepcidin promoter, at least in liver cells. ${ }^{45,46}$ Disturbance of the GATA-4-binding site downregulates hepcidin expression in response to IL-6, but not to BMP6. ${ }^{45}$ Future studies should address the question whether GATA-4 has a similar binding site in cardiac myocytes. Nonetheless, by serving as an antiapoptotic signal cardiac hepcidin can protect cardiomyocytes from excessive apoptosis in the heart, and this is crucial because apoptosis is linked to the emergence of HF.

Cardiac hepcidin has been proposed as a mediator of cardiac hypertrophy in Dahl salt-sensitive rats. ${ }^{47}$ High-salt diet in these rats causes hypertension and compensatory hypertrophy, whereas knockdown of cardiac hepcidin attenuates cardiac hypertrophy. ${ }^{47}$ Role of cardiac hepcidin in cardiac hypertrophy can be explained from results obtained with transgenic mice with dilated cardiomyopathy ${ }^{48}$ Hepcidin in these mice is downregulated, while restoring its expression induces significant cardiac hypertrophy (Table 1). This compensatory hypertrophy does not affect cardiac ultrastructure and it is associated with reduced interstitial fibrosis. ${ }^{48}$ Still, it is unclear what drives cardiac hepcidin expression in Dahl salt-sensitive rats, with iron load being an unlikely candidate. ${ }^{47}$ In addition, it would be interesting to understand the relationship between chronic overexpression of cardiac hepcidin and the maintenance of the functionally beneficial cardiac hypertrophy, as Dahl salt-sensitive rats eventually develop HF. ${ }^{47,48}$

Models of transgenic mice have revealed the molecular pathways that are associated with improved cardiac function during cardiac hepcidin expression. This improvement is accompanied by increased phosphorylation of extracellular signal-regulated kinase 1/2 (ERK1/2) levels in cardiomyocytes, which suggests that the beneficial role of hepcidin in this setting is at least partially linked with recovering of intracellular signaling through the ERK pathway. ${ }^{48}$ ERK proteins are important signaling factors that control cardiac hypertrophy. ${ }^{49}$ What is more important, ERK2 has been shown to protect cardiac muscle against reperfusion injury. ${ }^{49}$ It would be interesting to analyze levels of ERK proteins in heart-specific hepcidin knockout models during reperfusion injury. In this way we could unveil molecular mediators of cardiac hepcidin actions in protecting the cardiac muscle during reperfusion injury.

It has to be mentioned that this is not the first time that hepcidin is linked with beneficial actions outside the classical role in controlling iron load in cells. Hepcidin has been proposed to have a direct antifibrotic effect in the liver by keeping the quiescent status of hepatic stellate cells. ${ }^{50}$ In the biliary system, it acts as a potent antimicrobial agent and a marker of acute stress situations. ${ }^{51}$ It remains to be seen whether hepcidin actions are outside the scope of iron regulation in the heart as well.

\section{Cardiac Stress is Regularly Associated with Increased Hepcidin Expression}

There is a pattern of increased hepcidin expression in organs under stress, and this is the case with heart as well. In the early phase of myocardial infarction (MI) and in myocarditis, cardiac hepcidin expression is increased significantly. ${ }^{52}$ Hepcidin upregulation under these conditions is related to inflammatory markers, and this is especially the case with myocarditis. It is interesting to notice that, in $\mathrm{MI}$, the rise in levels of hepcidin is not accompanied with an increase in levels of cardiac HJV, reflecting an HJV-independent upregulation of cardiac hepcidin in MI. ${ }^{53}$ This increase in hepcidin expression is in correlation with IL-6 levels in the heart, which is not surprising, as IL-6 is a known inflammatory signal that induces hepcidin expression.[51] Another clue that hepcidin is increased in stress situations in the heart is its correlation with B-type natriuretic peptide, which has important cardioprotective functions during heart stress. ${ }^{54,55}$ It is interesting to notice that, in models of heart hypoxia, cardiac hepcidin is upregulated, which is in contrast with downregulation of liver hepcidin seen during systemic hypoxia. ${ }^{43}$ This might mean that hypoxia in the heart induces hepcidin upregulation as a protective mean in heart homeostasis. This role could be related to anti-apoptotic actions of cardiac hepcidin. During cardiac iron overload cardiac hepcidin protects cardiomyocytes against apoptosis. ${ }^{44}$ Another protective role of cardiac hepcidin in hypoxia/ ischemia is the increase in ferritin levels. Hepcidin increases ferritin, which sequesters excess iron that might cause oxidative damage in cardiomyocytes not only during ischemia, but also during reperfusion. ${ }^{30,31,56}$

Cardiac iron excess is one of the complications of $\mathrm{HH}$. High iron load (determined by levels of ferritin above $1000 \mu \mathrm{g})$ in this condition is associated with significant myocardial siderosis and heart dysfunction. However, this paradigm has been rivaled by studies from which we have learnt that cellular injury could be a more important factor than iron signaling in inducing cardiac hepcidin expression. ${ }^{55}$ 
This would be why hepcidin expression is observed in remote myocardium in the setting of MI without an accompanied increase in ferritin depots. ${ }^{53,55}$ On the other hand, it has to be mentioned that the lack of correlation between cardiac ferritin and hepcidin in rat models of cardiac injury is additionally complicated by the existence of sex differences in the regulation of iron metabolism. ${ }^{57}$

During cardiac injury, levels of BMP6 are upregulated in synchrony with levels of cardiac hepcidin, but there exists no correlation between these two parameters, as the one observed in the liver. ${ }^{55}$ BMP6 is expressed in the heart and it is involved in cardiogenesis. ${ }^{58}$ Its increase during cardiac injury has been proposed as an important downstream signal that is a part of a protective mechanism, which results in cardiac hypertrophy, increased vascularity, and decreased fibrosis. ${ }^{59}$ This reaction preserves heart function and reduces the risk of HF. On the other hand, secretion of BMP6 during cardiac injury, similar to the liver, is realized in a paracrine manner, that is from endothelial cells of coronary arteries. ${ }^{59}$ Recent observations of increased BMP6 levels in HF are in concert with these finding, and they suggest a reactive response to cardiac injury. ${ }^{60}$ In conclusion, the release of hepcidin and BMP6 during cardiac injury might serve as part of an orchestrated cardiac response to protect the myocardial function. Still, molecular mediators of BMP6 expression in the heart are unknown, whereas the relation of BMP6 with cardiac hepcidin remains to be elucidated.

TFR2, as it was previously mentioned, is a positive regulator of hepcidin expression. It exists in two isoforms, one of which is expressed in the heart and its downregulation is associated with heart protection during ischemia and reperfusion injury. ${ }^{61}$ The downregulation of cardiac TFR2 is accompanied by increased ferritin and decreased FPN expression, which is similar to the effect of hepcidin. ${ }^{61,62}$ However, data suggest that this action of TFR2-beta is realized through direct transcriptional modification of FPN, ${ }^{62}$ which would make TFR2-beta an unlikely signaling candidate in inducing cardiac hepcidin during reperfusion injury. It is interesting to notice the dichotomy between hepcidin and TFR2-beta in cardiac cells; myocardial injury increases expression of cardiac hepcidin and TFR2-beta, but hepcidin protects cardiomyocytes in this setting, whereas TFR2-beta overexpression has deleterious effect on heart function. It would seem that tipping the balance in favor of cardiac hepcidin expression would offer increased preservation of myocardial function during ischemia/reperfusion injury. This hypothesis needs to be validated by further research.

\section{ROLE OF SYSTEMIC HEPCIDIN IN HEART HOMEOSTASIS}

The most prevalent genetic condition characterized with disturbance in liver hepcidin production and action is HH. It encompasses several distinct forms of the disease, of which juvenile $\mathrm{HH}$ has a more malign course compared with classical HFE-HH. The reason for this outcome is that in juvenile $\mathrm{HH}$ hepcidin expression is disrupted more significantly than in HFE-HH, resulting in more severe organ iron load. ${ }^{13}$ One of the complications of $\mathrm{HH}$ is iron-load cardiomyopathy. Although the risk of developing organ damage in $\mathrm{HH}$ is low, cardiomyopathy in $\mathrm{HH}-\mathrm{HFE}$ patients is still higher than in non-HFE-HH patients. ${ }^{63}$ This risk is mostly confined to patients with high levels of iron load, which is confirmed by the observation that myocardial siderosis is present in $0 \%$ of patients with levels of ferritin $<1000 \mu \mathrm{g} / \mathrm{l}$. However, when levels of ferritin are $>1000 \mu \mathrm{g} / \mathrm{l}$, myocardial siderosis increases markedly and it is present in $1 / 3$ of patients. ${ }^{64}$ Myocardial siderosis causes oxidative damage and resultant heart dysfunction. ${ }^{65}$

It is believed that in conditions with iron excess, iron enters myocardial cells through L-type calcium channels (LTCC). ${ }^{66}$ When iron depots get overloaded, the levels of free iron go up and lead to the formation of ROS. ${ }^{65}$ ROS causes disruption of the integrity of many cellular structures, which will eventually lead to heart dysfunction. Preventing heart dysfunction in HFE-HH can be reversed with phlebotomy and iron chelator therapy. ${ }^{67,68}$ Data suggest that LTCCs are not the only way of iron entry in myocardial cells. In thalassemic patients, iron entry in cardiomyocytes can be prevented by T-type calcium channel (TTCC) blockers, which suggests a role for TTCC for iron entry in cardiac cells. ${ }^{69}$ This is the reason why the dual LTCC and TTCC blocker efonidipine has additional benefits compared with verapamil, which is a sole LTCC blocker. ${ }^{70}$

In FA iron overload is also frequently present and is related to mitochondrial dysfunction. ${ }^{71,72}$ There is an increasing evidence that specific cellular compartmentalization of iron in mitochondria accompanied with cytosolic iron deficiency is behind heart dysfunction observed in FA. ${ }^{71,72}$ Although iron supplementation does limit cardiac hypertrophy in models of mice with FA, tackling mitochondrial iron overload should be included in therapeutic strategies under this condition. ${ }^{73,74}$ Serum hepcidin in models of mice with FA are increased, coinciding with an increase in expression of HJV, which is a major positive regulator of hepcidin. ${ }^{73}$ It seems that levels of liver hepcidin in FA occur in response to increased iron load in the liver.

Role of liver hepcidin has been studied in conditions associated with iron deficiency as well, such as HF. Anemia in HF patients is frequent and weakly related to myocardial iron content, which is reduced in these patients. ${ }^{75}$ It is interesting to notice that, although mitochondrial dysfunction is present in HF patients with myocardial iron deficiency (MID), anemia is not correlated with mitochondrial dysfunction. ${ }^{75}$ This suggests that mitochondrial dysfunction in cardiomyocytes is not directly related to MID, but is probably caused by the underlying pathophysiological process related to cardiac injury that eventually progresses to HF. For example, in ischemic heart disease there is an increase in mitochondrial iron, which causes mitochondrial dysfunction through oxidative stress. ${ }^{76}$ Reduction of mitochondrial iron with iron chelators and overexpression of mitochondrial iron exporters 


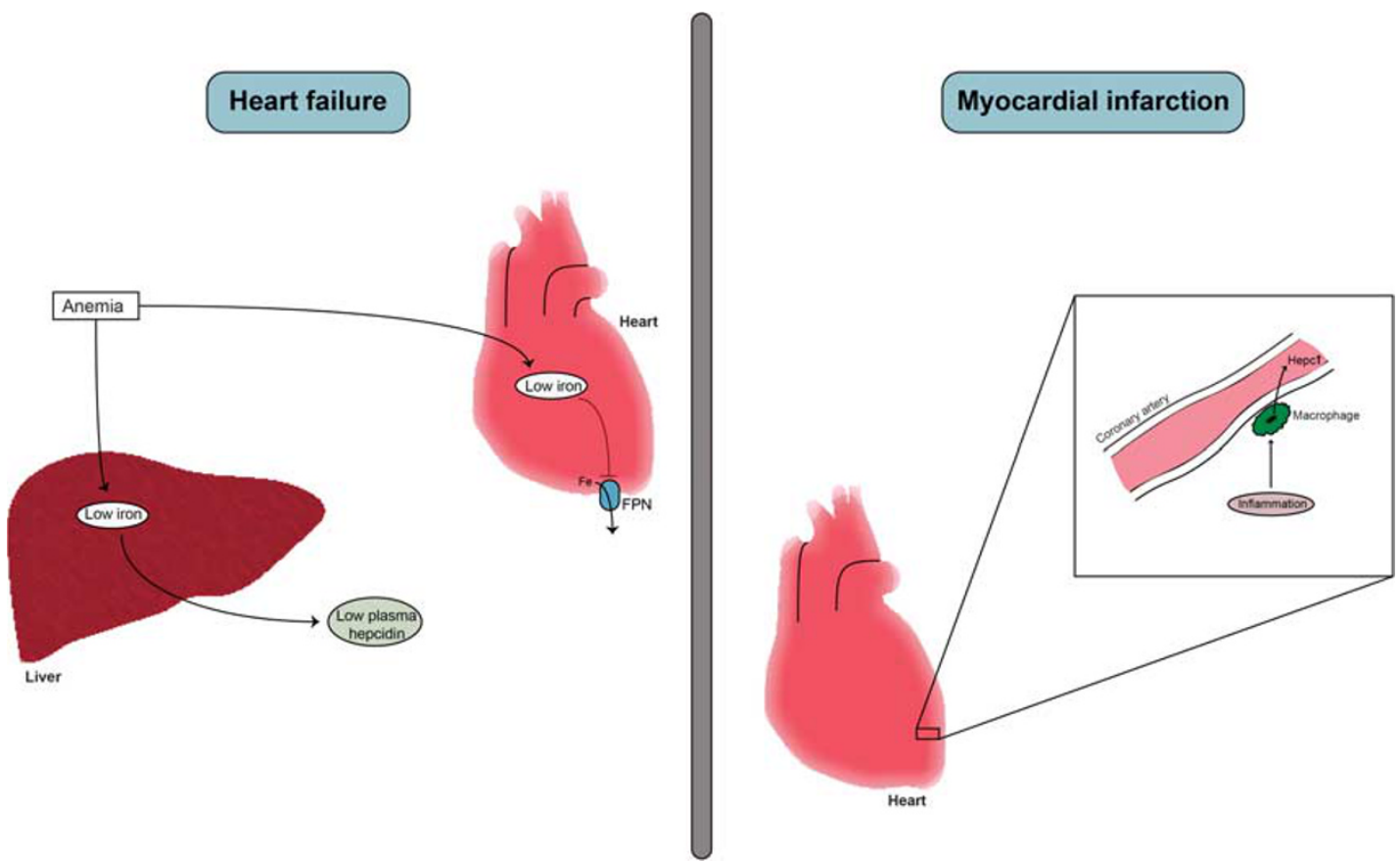

Figure 3 Role of liver (systemic) hepcidin in heart disease. In heart failure levels of systemic hepcidin are low in the advanced stages of disease. These levels are in response to anemia and resultant low iron depots inside hepatocytes. In the heart, iron deficiency causes downregulation of FPN, through which cardiomyocytes try to keep their iron depots. In myocardial infarction levels of systemic hepcidin are high. The source of increased hepcidin expression is activated macrophages. FPN, ferroportin.

protects cardiomyocytes against damage caused by oxidative stress. ${ }^{76}$

On the other hand, MID is associated with certain aspects of mitochondrial dysfunction, such as reduction of the activity of citric acid cycle enzymes and ROS-protecting enzymes, some of which use iron as a cofactor. ${ }^{75}$ One of these enzymes is catalase, and its overexpression in models of mice with HF restores mitochondrial functionality. ${ }^{77}$ The observed changes mean that MID might worsen the already present mitochondrial dysfunction in HF through specific mechanisms. In-line with the associations of MID with heart dysfunction are also results from experiments with mice lacking TFR1. These mice develop serious heart damage and mitochondrial dysfunction, which can be reversed by early aggressive iron supplementation. ${ }^{78}$ Similarly, reduced activity of IREs and loss of IRPs has been observed in advanced HF, which is important, as IREs and IRPs control cellular iron availability. ${ }^{38}$ Furthermore, loss of IRPs increases the risk of HF after MI. ${ }^{38}$ It has to be mentioned that loss of IRPs is associated with an increase in mitochondrial ferritin, which might indicate preferential iron transport into mitochondria. ${ }^{38}$ These observations show that mitochondria are sensitive to iron deficiency and iron overload, both of which can cause serious mitochondrial dysfunction, although there are suggestions that iron overload is a more prominent etiological factor in mitochondrial dysfunction. ${ }^{79}$

Anemia is frequently present in HF patients, but hepcidin does not seem to be an important mediator of anemia in this condition. ${ }^{80,81}$ Hepcidin in HF is correlated with iron stores, erythropoiesis, and liver congestion. ${ }^{36,82}$ This suggests a preserved hepcidin expression in response to iron status and erythropoietic drive. Hepcidin expression is induced in liver congestion even in the setting of anemia because of high levels of IL-6. ${ }^{82}$ According to Jankowska et al., levels of hepcidin in patients with HF start to drop with increase in HF stage. ${ }^{36}$ In addition, according to this study, low levels of hepcidin are predictors of mortality. Lower levels of hepcidin observed in advanced HF are probably a result of the suppression of hepcidin in response to the degree of iron deficiency (Figure 3). Furthermore, higher baseline hepcidin levels are predictors of nonresponsiveness to oral iron therapy in anemia in HF according to IRONOUT-HF-randomized clinical trial. ${ }^{83}$ Similarly, in patients with iron-deficient anemia higher levels of hepcidin are related with nonresponsiveness to oral iron. ${ }^{84}$ Decreased gastrointestinal absorption of iron in HF means that IV iron is more likely to correct anemia in $\mathrm{HF}^{85}$ Indeed, the latest results from EFFECT-HF trial seem to confirm these suggestions. ${ }^{86}$

CKD can cause increased levels of liver hepcidin because of impaired glomerular filtration and inflammation. ${ }^{87}$ However, even in these patients, iron deficiency lowers liver hepcidin expression, confirming the crucial role of iron status in hepcidin regulation. ${ }^{88}$ Systemic hepcidin levels can serve as a therapeutic target in CKD because according to the study from Hsieh et al., low levels of serum hepcidin in CKD are negative predictors of left ventricular hypertrophy. ${ }^{88}$ Still, 
authors of this study did not evaluate the mechanistic links between low hepcidin and cardiac hypertrophy, which would shed light on the role of systemic hepcidin in heart damage during CKD.

More evidence comes from chronic hemodialysis patients with diabetic nephropathy, where high serum hepcidin is independently associated with common carotid artery intima media thickness, which is a marker of atherosclerosis. ${ }^{89}$ Similar results in hemodialysis patients have been observed by other authors as well. ${ }^{90}$ Furthermore, high levels of hepcidin in hemodialysis patients were shown to be a risk factor for fatal and non-fatal cardiovascular events. ${ }^{91}$ High hepcidin in this condition is probably caused by inflammatory stimuli, which is enforced by the existence of high correlation between hepcidin and inflammatory markers such as hsCRP, and IL-6. ${ }^{89}$ In addition, under conditions characterized with high levels of inflammation, like rheumatoid arthritis, increased risk of atherosclerosis is related with high levels of hepcidin, ${ }^{92}$ whereas in Kawasaki disease higher serum hepcidin levels are associated with coronary atherosclerosis and inappropriate treatment response to therapy. ${ }^{93}$

However, in general population without the presence of high levels of inflammation serum hepcidin is not associated with incident cardiovascular disease. ${ }^{94}$ On the other hand, in postmenopausal healthy women, hepcidin is related to atherosclerosis. ${ }^{95}$ It is interesting to notice that in the study by Galesloot et al. ${ }^{95}$ hepcidin levels were higher compared with those described in the study by Pechlaner et al. ${ }^{94}$ where only $2 \%$ of patients showed high levels of hepcidin. This might imply that higher baseline hepcidin levels can serve as a pathogenic risk co-factor in atherosclerosis. Furthermore, the most significant associations between serum hepcidin levels and plaque presence in the study by Galesloot et al. were observed with higher quartiles of hepcidin values, whereas in men only the highest quartile showed an association with atherosclerosis. ${ }^{95}$ In conclusion, serum hepcidin can serve convincingly as a marker of cardiac damage and atherosclerosis in specific populations like patients on hemodialysis, ${ }^{89-91}$ patients with higher baseline levels of hepcidin (especially women), ${ }^{95}$ but also in high-risk patients with metabolic alterations. ${ }^{96}$ This strengthens the notion that hepcidin is at least a co-contributing factor in plaque formation and destabilization, especially when other risk factors are present. This idea is further enforced by the observation that serum hepcidin can serve as an independent marker of cardiovascular events in hemodialysis patients independently of inflammation.[90] In addition, hepcidin levels might be related with the severity of plaque destabilization, which is in accordance with higher levels of iron observed in the plaques of symptomatic patients compared with non-symptomatic patients. ${ }^{97}$ Interestingly, levels of iron protein importers, such as TFR1, are also increased in plaques of symptomatic patients and are associated with plaque instability. ${ }^{98}$ In addition, it has been suggested that $\mathrm{HH}$ patients seem to be protected from atherosclerosis, which can be explained by the lower levels of serum hepcidin observed in this condition. ${ }^{99-101}$

In vitro data show that macrophages can cause plaque instability by increasing hepcidin production. ${ }^{102}$ What is more, suppression of liver hepcidin through BMPR inhibitors in models of mice with atherosclerosis results in increased cholesterol efflux from macrophages and decreased formation of foam cells. ${ }^{103}$ This action of hepcidin was related with its effects on FPN, which might not seem surprising as reduction of intracellular iron in plaque macrophages increases cholesterol efflux through upregulation of $\mathrm{ABC}$ transporters. ${ }^{104}$ It should be noted that the observed effect of BMPR inhibitors might not occur solely as a result of hepcidin suppression but also because of direct consequences of inhibition of BMP signaling, which has been observed by earlier studies. ${ }^{105}$ Furthermore, inhibition of BMP signaling reduces levels of inflammation, ${ }^{105}$ which, as studies show, is an important factor in promoting hepcidin plaque destabilization. ${ }^{89-93}$ This might be the reason why Kautz et al. did not observe a significant role of FPN mutations (unresponsive to hepcidin action) in progression of atherosclerosis. ${ }^{106}$ This study was characterized with low levels of inflammation and without a significant rise in IL-6 levels, which is a known inducer hepcidin expression through the JAK/STAT pathway. ${ }^{15}$ It would be interesting to examine the interaction between increasing levels of hepcidin and inflammation in models of atherosclerosis in relation to plaque stability.

Changes in systemic hepcidin levels have been detected in acute MI. There have been reports that levels of hepcidin-20 and hepcidin-25 are increased in MI. ${ }^{107}$ However, this study could not resolve the issue of tissue origin of increased hepcidin levels. The dilemma might have been solved by the work from Sasai et al., in which higher levels of hepcidin-25 and hepcidin-20 were detected in aspirate samples from coronary arteries compared with peripheral blood samples. ${ }^{102}$ Accompanied in vitro experiments from this study detected macrophages from atherosclerotic plaques as the cellular 'culprits' of increased hepcidin levels. This is an interesting observation because it shows that hepcidin from other sources than liver can contribute to total levels of systemic hepcidin. More importantly, according to this study, hepcidin produced by macrophages was related to endothelial damage and plaque instability. This would make increased serum levels of hepcidin a negative prognostic factor in MI.

\section{CONCLUDING REMARKS AND CLINICAL APPLICATIONS}

Latest research has revealed an interesting picture about the role of hepcidin in heart homeostasis. This role seems to be crucial considering the marked effect of cardiac hepcidin deficiency in heart function. Under specific conditions, like iron deficiency and hypoxia, regulation of cardiac hepcidin is different from liver hepcidin, suggesting a distinct role of both hepcidins in heart homeostasis. It is interesting to notice that there are suggestions that protective effects of hepcidin in 
heart function might span outside the classical iron-regulatory role of this peptide. These suggestions should be examined in further detail by future studies.

Data suggest that heart homeostasis is affected more markedly with loss of cardiac FPN and hepcidin compared with the loss of systemic FPN and hepcidin. Loss of cardiac hepcidin is directly related to cardiac iron deficiency, which can be reversed by restoring cardiac hepcidin expression. ${ }^{28}$ As iron supplementation has been consistently shown to restore mitochondrial function in MID, while preferential mitochondrial ROS inhibition offers cardioprotective effects, then this means that restoring cardiac hepcidin expression in conjunction with mitochondrial ROS inhibition could prove to be a new therapeutic strategy in HF. However, we must not forget the complex nature of mitochondrial dysfunction in HF that should be taken into account when using target therapy. For example, ischemic etiology of HF compared with nonischemic etiology is more prominently related to mitochondrial dysfunction and mitochondrial oxidative stress. ${ }^{108}$ In addition, hormonal sex differences have been proposed as a factor that can affect intracellular ROS production. ${ }^{37}$ Finally, there is experimental evidence from mice that age-related differences in mitochondrial biochemistry can affect treatment strategies in heart dysfunction. ${ }^{109}$

Encouraging news comes from manipulating cardiac hepcidin expression in models of transgenic mice with dilated cardiomyopathy as well. In these mice local hepcidin expression protects from fibrosis and improves heart function. ${ }^{48}$ Still, there remain hurdles to this strategy in clinical practice, the main one of which would be specific induction of hepcidin in heart cardiomyocytes without affecting systemic hepcidin levels.

The role of cardiac hepcidin could be beneficial in cardiac ischemia as well. There are suggestions that increased cardiac hepcidin protects cardiomyocytes from iron toxicity, but also by reducing extracellular iron pool. ${ }^{52,53}$ Unfortunately, knockdown and overexpression of cardiac hepcidin has not been studied in cardiac ischemia, which would help us elucidate the role of cardiac hepcidin in this setting.

In FA local anti-hepcidin therapy can be beneficial as increased hepcidin production from cardiac inflammatory infiltrate is related to the pathogenesis of FA, although this hypothesis needs to be validated by future research.

Although cardiac hepcidin has an important role in maintaining cellular homeostasis, systemic hepcidin has an important role as well, which is seen during chronic loss of liver hepcidin production and action. This is confirmed by increased risk of cardiomyopathy observed in conditions with high iron load, such as $\mathrm{HH}$. It has to be mentioned that in $\mathrm{HH}$ cardiac iron overload is less frequently observed than liver iron overload. The accumulation of iron in noncardiomyocytes during systemic hepcidin deficiency and the autonomous role of cardiac hepcidin in controlling cardiac iron depots might be the reason for this observation, although it remains to be confirmed by studies that will evaluate cardiac hepcidin expression in HH. Unfortunately, the effects of experimental overexpression of liver hepcidin and treatment with synthetic hepcidins have been studied only in terms of correction of liver iron load. ${ }^{10,111}$ Comparisons between overexpression of systemic vs cardiac hepcidin in acute and chronic setting can yield us the necessary results about the importance of recovering hepcidin signaling in heart function. As liver hepcidin has a role in heart homeostasis, it would be interesting to compare the efficacy of iron reduction therapy between iron chelators or phlebotomy and hepcidin therapy during cardiac iron overload. Iron chelation or phlebotomy does not solve the problem of hepcidin loss; therefore, it is probable that recuperating hepcidin levels through hepcidin agonists could potentially increase the therapeutic success in cardiac iron overload.

Although iron load through hepcidin deficiency is a known factor in heart dysfunction, iron deficiency is also important, as it is related to one of the most prevalent heart conditions, which is HF. Many studies have shown that iron deficiency dysregulates cardiac cellular biochemistry, which is why we observe low levels of serum hepcidin in HF. Low levels of hepcidin seem to serve as a counteractive mechanism that fights iron deficiency by securing more iron supply from enterocytes and macrophages into the plasma. Therefore, restoring liver hepcidin levels in this setting might be counterproductive. It is interesting to notice that drugs used in cardiac pharmacotherapy, such as spironolactone and heparins, are known suppressors of liver hepcidin, although it is not known whether they can affect iron metabolism in HF. ${ }^{112,113}$

Increased serum hepcidin levels in MI might be deleterious for cardiac function. This happens because increased serum hepcidin in this setting, at least partially, originates from activated macrophages of the atherosclerotic arteries, where excess hepcidin induces plaque instability, probably through increased oxidative stress. Indeed, hepcidin overexpression in models of mice with atherosclerotic lesions induces plaque instability, whereas hepcidin knockdown reverses these changes. ${ }^{114}$ These observations can serve future therapeutic regimens in atherosclerosis.

\section{DISCLOSURE/CONFLICT OF INTEREST}

The author declares no conflict of interest.

1. Coffey R, Ganz T. Iron homeostasis-an anthropocentric perspective. J Biol Chem 2017;R117:781823.

2. Cairo G, Recalcati S. Iron-regulatory proteins: molecular biology and pathophysiological implications. Expert Rev Mol Med 2007;9:1-13.

3. Bogdan AR, Miyazawa M, Hashimoto K, et al. Regulators of iron homeostasis: new players in metabolism, cell death, and disease. Trends Biochem Sci 2016;41:274-286.

4. Gao G, Chang Y-Z. Mitochondrial ferritin in the regulation of brain iron homeostasis and neurodegenerative diseases. Front Pharmacol 2014;5:19.

5. Addo L, Ikuta K, Tanaka $\mathrm{H}$, et al. The three isoforms of hepcidin in human serum and their processing determined by liquid 
chromatography-tandem mass spectrometry (LC-tandem MS). Int J Hematol 2016;103:34-43.

6. Nicolas G, Bennoun M, Devaux I, et al. Lack of hepcidin gene expression and severe tissue iron overload in upstream stimulatory factor 2 (USF2) knockout mice. Proc Natl Acad Sci USA 2001;98: 8780-8785.

7. Andriopoulos Jr B , Corradini E, Xia Y, et al. BMP6 is a key endogenous regulator of hepcidin expression and iron metabolism. Nat Genet 2009;41:482-487.

8. Wallace DF, Summerville L, Crampton EM, et al. Combined deletion of $\mathrm{Hfe}$ and transferrin receptor 2 in mice leads to marked dysregulation of hepcidin and iron overload. Hepatology 2009;50:1992-2000.

9. Canali S, Zumbrennen-Bullough KB, Core $A B$, et al. Endothelial cells produce bone morphogenetic protein 6 required for iron homeostasis in mice. Blood 2017;129:405-414.

10. Steinbicker AU, Bartnikas TB, Lohmeyer LK, et al. Perturbation of hepcidin expression by BMP type I receptor deletion induces iron overload in mice. Blood 2011;118:4224-4230.

11. Babitt JL, Huang FW, Wrighting DM, et al. Bone morphogenetic protein signaling by hemojuvelin regulates hepcidin expression. Nat Genet 2006;38:531-539.

12. Corradini E, Meynard D, Wu Q, et al. Serum and liver iron differently regulate the bone morphogenetic protein 6 (BMP6)-SMAD signaling pathway in mice. Hepatology 2011;54:273-284.

13. Camaschella C. Understanding iron homeostasis through genetic analysis of hemochromatosis and related disorders. Blood 2005;106: 3710-3717.

14. Nemeth E, Rivera S, Gabayan V, et al. IL-6 mediates hypoferremia of inflammation by inducing the synthesis of the iron regulatory hormone hepcidin. J Clin Invest 2004;113:1271-1276.

15. Wrighting DM, Andrews NC. Interleukin-6 induces hepcidin expression through STAT3. Blood 2006;108:3204-3209.

16. Besson-Fournier C, Gineste A, Latour C, et al. Hepcidin upregulation by inflammation is independent of Smad1/5/8 signaling by activin B. Blood 2017;129:533-536.

17. Canali $S$, Core $A B$, Zumbrennen-Bullough $K B$, et al. Activin B induces noncanonical SMAD1/5/8 signaling via BMP type I receptors in hepatocytes: evidence for a role in hepcidin induction by inflammation in male mice. Endocrinology 2016;157:1146-1162.

18. Kautz L, Jung G, Valore EV, et al. Identification of erythroferrone as an erythroid regulator of iron metabolism. Nat Genet 2014;46: 678-684.

19. Bacchetta J, Zaritsky JJ, Sea JL, et al. Suppression of iron-regulatory hepcidin by vitamin D. J Am Soc Nephrol 2014;25:564-572.

20. Lehtihet $M$, Bonde $Y$, Beckman $L$, et al. Circulating hepcidin-25 is reduced by endogenous estrogen in humans. PLoS ONE 2016;11: e0148802.

21. Liu Q, Davidoff O, Niss K, et al. Hypoxia-inducible factor regulates hepcidin via erythropoietin-induced erythropoiesis. J Clin Invest 2012;122:4635-4644.

22. Nemeth E, Tuttle MS, Powelson J, et al. Hepcidin regulates cellular iron efflux by binding to ferroportin and inducing its internalization. Science 2004;306:2090-2093.

23. Qiao B, Sugianto $P$, Fung $E$, et al. Hepcidin-induced endocytosis of ferroportin is dependent on ferroportin ubiquitination. Cell Metab 2012;15:918-924.

24. Ganz T, Nemeth E. Hepcidin and disorders of iron metabolism. Annu Rev Med 2011;62:347-360.

25. Chen QX, Song SW, Chen QH, et al. Silencing airway epithelial cellderived hepcidin exacerbates sepsis-induced acute lung injury. Crit Care 2014;18:470.

26. Schwarz $P$, Kübler JAM, Strnad $P$, et al. Hepcidin is localised in gastric parietal cells, regulates acid secretion and is induced by Helicobacter pylori infection. Gut 2012;61:193-201.

27. Tesfay L, Clausen KA, Kim JW, et al. Hepcidin regulation in prostate and its disruption in prostate cancer. Cancer Res 2015;75: 2254-2263.

28. Lakhal-Littleton S, Wolna M, Chung YJ, et al. An essential cellautonomous role for hepcidin in cardiac iron homeostasis. Elife 2016;5:867-878.

29. Krause A, Neitz S, Mägert H-J, et al. LEAP-1, a novel highly disulfidebonded human peptide, exhibits antimicrobial activity. FEBS Lett 2000;480:147-150.
30. Ge XH, Wang Q, Qian ZM, et al. The iron regulatory hormone hepcidin reduces ferroportin 1 content and iron release in $\mathrm{H} 9 \mathrm{C} 2$ cardiomyocytes. J Nutr Biochem 2009;20:860-865.

31. Lakhal-Littleton S, Wolna M, Carr CA, et al. Cardiac ferroportin regulates cellular iron homeostasis and is important for cardiac function. Proc Natl Acad Sci USA 2015;112:3164-3169.

32. Fang $X$, Wang $H, A n P$, et al. Cardiomyocyte-specific deletion of ferroportin using MCK-Cre has no apparent effect on cardiac iron homeostasis. Int J Cardiol 2015;201:90-92.

33. Adams PC, Speechley $M$, Kertesz AE. Long-term survival analysis in hereditary hemochromatosis. Gastroenterology 1991;101:368-372.

34. Pietrangelo A. The ferroportin disease. Clin Liver Dis 2014;3:98-100.

35. Qian ZM, Chang YZ, Leung G, et al. Expression of ferroportin1, hephaestin and ceruloplasmin in rat heart. Biochim Biophys Acta 2007;1772:527-532.

36. Jankowska EA, Malyszko J, Ardehali $\mathrm{H}$, et al. Iron status in patients with chronic heart failure. Eur Heart J 2013;34:827-834.

37. Shapiro JS, Chang $\mathrm{H}$, Ardehali $\mathrm{H}$. Iron and sex cross paths in the heart. J Am Heart Assoc 2017;6:e005459.

38. Haddad S, Wang Y, Galy B, et al. Iron-regulatory proteins secure iron availability in cardiomyocytes to prevent heart failure. Eur Heart J 2016;98:ehw333.

39. Gagliardo B, Kubat N, Faye A, et al. Pro-hepcidin is unable to degrade the iron exporter ferroportin unless maturated by a furin-dependent process. J Hepatol 2009;50:394-401.

40. Susan-Resiga D, Essalmani R, Hamelin J, et al. Furin is the major processing enzyme of the cardiac-specific growth factor bone morphogenetic protein 10. J Biol Chem 2011;286:22785-22794.

41. Koeppen $A H$, Ramirez RL, Becker $A B$, et al. The pathogenesis of cardiomyopathy in Friedreich ataxia. PLoS ONE 2015;10:e0116396.

42. Ramirez RL, Qian J, Santambrogio P, et al. Relation of cytosolic iron excess to cardiomyopathy of Friedreich's ataxia. Am J Cardiol 2012;110:1820-1827.

43. Merle U, Fein E, Gehrke SG, et al. The iron regulatory peptide hepcidin is expressed in the heart and regulated by hypoxia and inflammation. Endocrinology 2007;148:2663-2668.

44. Hsieh $\mathrm{Y}-\mathrm{P}$, Huang $\mathrm{C}-\mathrm{H}$, Lee $\mathrm{C}-\mathrm{Y}$, et al. Silencing of hepcidin enforces the apoptosis in iron-induced human cardiomyocytes. J Occup Med Toxicol 2014;9:1-8.

45. Island $\mathrm{M}-\mathrm{L}$, Fatih $\mathrm{N}$, Leroyer $\mathrm{P}$, et al. GATA-4 transcription factor regulates hepatic hepcidin expression. Biochem J 2011;437:477-482.

46. Suzuki YJ, Evans T. Regulation of cardiac myocyte apoptosis by the GATA-4 transcription factor. Life Sci 2004;74:1829-1838.

47. Naito $\mathrm{Y}$, Hosokawa M, Sawada $\mathrm{H}$, et al. Hepcidin is increased in the hypertrophied heart of Dahl salt-sensitive rats. Int J Cardiol 2014;172: e45-e47.

48. Zhang L, Lu D, Zhang W, et al. Cardioprotection by Hepc1 in cTnTR141W transgenic mice. Transgenic Res 2012;21:867-878.

49. Mutlak $M$, Kehat I. Extracellular signal-regulated kinases $1 / 2$ as regulators of cardiac hypertrophy. Front Pharmacol 2015;6:149.

50. Han CY, Koo JH, Kim SH, et al. Hepcidin inhibits Smad3 phosphorylation in hepatic stellate cells by impeding ferroportinmediated regulation of Akt. Nat Commun 2016;7:13817.

51. Strnad P, Schwarz P, Rasenack MCD, et al. Hepcidin is an antibacterial, stress-inducible peptide of the biliary System. PLoS ONE 2011;6: e16454.

52. Isoda $M$, Hanawa $H$, Watanabe $R$, et al. Expression of the peptide hormone hepcidin increases in cardiomyocytes under myocarditis and myocardial infarction. J Nutr Biochem 2010;21:749-756.

53. Simonis G, Mueller K, Schwarz $P$, et al. The iron-regulatory peptide hepcidin is upregulated in the ischemic and in the remote myocardium after myocardial infarction. Peptides 2010;31: 1786-1790.

54. Saito Y. Roles of atrial natriuretic peptide and its therapeutic use. J Cardiol 2010;56:262-270.

55. van Breda GF, Bongartz LG, Zhuang W, et al. Cardiac hepcidin expression associates with injury independent of iron. Am J Nephrol 2016;44:368-378.

56. Pucheu S, Coudray C, Tresallet N, et al. Effect of iron overload in the isolated ischemic and reperfused rat heart. Cardiovasc Drugs Ther 1993;7:701-711

57. Kong W-N, Niu Q-M, Ge L, et al. Sex differences in iron status and hepcidin expression in rats. Biol Trace Elem Res 2014;160:258-267. 
58. Kim RY, Robertson EJ, Solloway MJ. Bmp6 and Bmp7 are required for cushion formation and septation in the developing mouse heart. Dev Biol 2001;235:449-466.

59. Korf-Klingebiel M, Kempf T, Schlüter K-D, et al. Conditional Transgenic expression of fibroblast growth factor 9 in the adult mouse heart reduces heart failure mortality after myocardial infarction. Circulation 2011;123:504-514.

60. Banach J, Gilewski W, Słomka A, et al. W. Bone morphogenetic protein 6-a possible new player in pathophysiology of heart failure. Clin Exp Pharmacol Physiol 2016;43:1247-1250.

61. Boero M, Pagliaro P, Tullio F, et al. A comparative study of myocardial molecular phenotypes of two tfr $2 \beta$ null mice: role in ischemia/ reperfusion. BioFactors 2015;41:360-371.

62. Roetto A, Di Cunto F, Pellegrino RM, et al. Comparison of 3 Tfr2deficient murine models suggests distinct functions for Tfr2- and Tfr2- isoforms in different tissues. Blood 2010;115:3382-3389.

63. Pietrangelo A. Hereditary hemochromatosis - a new look at an old disease. N Engl J Med 2004;350:2383-2397.

64. Carpenter J-P, Grasso AE, Porter JB, et al. On myocardial siderosis and left ventricular dysfunction in hemochromatosis. J Cardiovasc Magn Reson 2013;15:24.

65. Das SK, Wang W, Zhabyeyev $\mathrm{P}$, et al. Iron-overload injury and cardiomyopathy in acquired and genetic models is attenuated by resveratrol therapy. Sci Rep 2016;5:18132.

66. Oudit GY, Sun H, Trivieri MG, et al. L-type Ca2+ channels provide a major pathway for iron entry into cardiomyocytes in iron-overload cardiomyopathy. Nat Med 2003;9:1187-1194.

67. Dabestani A, Child JS, Henze E, et al. Primary hemochromatosis: anatomic and physiologic characteristics of the cardiac ventricles and their response to phlebotomy. Am J Cardiol 1984;54:153-159.

68. Gabutti V, Piga A. Results of long-term iron-chelating therapy. Acta Haematol 1996;95:26-36.

69. Kumfu S, Chattipakorn S, Srichairatanakool S, et al. T-type calcium channel as a portal of iron uptake into cardiomyocytes of betathalassemic mice. Eur J Haematol 2011;86:156-166.

70. Kumfu S, Chattipakorn S, Chinda K, et al. T-type calcium channel blockade improves survival and cardiovascular function in thalassemic mice. Eur J Haematol 2012;88:535-548.

71. Huang ML-H, Becker EM, Whitnall $M$, et al. Elucidation of the mechanism of mitochondrial iron loading in Friedreich's ataxia by analysis of a mouse mutant. Proc Natl Acad Sci USA 2009;106:16381-16386.

72. Richardson DR, Huang ML-H, Whitnall $M$, et al. The ins and outs of mitochondrial iron-loading: the metabolic defect in Friedreich's ataxia. J Mol Med 2010;88:323-329.

73. Whitnall M, Suryo Rahmanto $\mathrm{Y}, \mathrm{ML}-\mathrm{H}$ Huang, et al. Identification of nonferritin mitochondrial iron deposits in a mouse model of Friedreich ataxia. Proc Natl Acad Sci USA 2012;109:20590-20595.

74. Anzovino A, Lane DJR, ML-H Huang, Richardson DR. Fixing frataxin: "ironing out" the metabolic defect in Friedreich's ataxia. $\mathrm{Br} J$ Pharmacol 2014;171:2174-2190.

75. Melenovsky V, Petrak J, Mracek T, et al. Myocardial iron content and mitochondrial function in human heart failure: a direct tissue analysis. Eur J Heart Fail 2017;19:522-530.

76. Chang $\mathrm{H}-\mathrm{C}, \mathrm{Wu} \mathrm{R}$, Shang $\mathrm{M}$, et al. Reduction in mitochondrial iron alleviates cardiac damage during injury. EMBO Mol Med 2016;8: 247-267.

77. Dai D-F, Hsieh EJ, Liu Y, et al. Mitochondrial proteome remodelling in pressure overload-induced heart failure: the role of mitochondrial oxidative stress. Cardiovasc Res 2012;93:79-88.

78. $\mathrm{Xu} \mathrm{W,} \mathrm{Barrientos} \mathrm{T,} \mathrm{Mao} \mathrm{L,} \mathrm{et} \mathrm{al.} \mathrm{Lethal} \mathrm{cardiomyopathy} \mathrm{in} \mathrm{mice}$ lacking transferrin receptor in the heart. Cell Rep 2015;13:533-545.

79. Chang H-C, Shapiro JS, Ardehali H. Getting to the "heart" of cardiac disease by decreasing mitochondrial iron. Circ Res 2016;119: 1164-1166.

80. Cleland JGF, Zhang J, Pellicori P, et al. Prevalence and outcomes of anemia and hematinic deficiencies in patients with chronic heart failure. JAMA Cardiol 2016;1:539.

81. Matsumoto $M$, Tsujino $T$, Lee-Kawabata $M$, et al. Iron regulatory hormone hepcidin decreases in chronic heart failure patients with anemia. Circ J 2010;74:301-306.

82. Suzuki T, Hanawa $\mathrm{H}$, Jiao $\mathrm{S}$, et al. Inappropriate expression of hepcidin by liver congestion contributes to anemia and relative iron deficiency. J Card Fail 2014;20:268-277.
83. Lewis GD, Malhotra $\mathrm{R}$, Hernandez AF, et al. Effect of oral iron repletion on exercise capacity in patients with heart failure with reduced ejection fraction and iron deficiency. JAMA 2017;317:1958-1966.

84. Bregman DB, Morris D, Koch TA, et al. Hepcidin levels predict nonresponsiveness to oral iron therapy in patients with iron deficiency anemia. Am J Hematol 2013;88:97-101.

85. McDonagh T, Macdougall IC. Iron therapy for the treatment of iron deficiency in chronic heart failure: intravenous or oral? Eur J Heart Fail 2015;17:248-262.

86. van Veldhuisen DJ, Ponikowski $P$, van der Meer $P$, et al. Effect of ferric carboxymaltose on exercise capacity in patients with chronic heart failure and iron deficiency. Circulation 2017; doi: 10.1161/ CIRCULATIONAHA.117.027497.

87. Wagner M, Ashby D. Hepcidin-a well-known iron biomarker with prognostic implications in chronic kidney disease. Nephrol Dial Transplant 2013;28:2936-2939.

88. Hsieh Y-P, Huang C-H, Lee C-Y, et al. Hepcidin-25 negatively predicts left ventricular mass index in chronic kidney disease patients. World J Nephrol 2013;2:38-43.

89. Li H, Feng S-J, Su L-L, et al. Serum hepcidin predicts uremic accelerated atherosclerosis in chronic hemodialysis patients with diabetic nephropathy. Chin Med J 2015;128:1351-1357.

90. Kali A, Yayar O, Erdogan B, et al. Is hepcidin-25 a predictor of atherosclerosis in hemodialysis patients? Hemodial Int 2016;20: 191-197.

91. van der Weerd NC, Grooteman MPC, Bots ML, et al. Hepcidin-25 is related to cardiovascular events in chronic haemodialysis patients. Nephrol Dial Transplant 2013;28:3062-3071.

92. Abdel-Khalek MA, El-Barbary AM, SA-M Essa, et al. Serum hepcidin: a direct link between anemia of inflammation and coronary artery atherosclerosis in patients with rheumatoid arthritis. J Rheumatol 2011;38:2153-2159.

93. Kuo H-C, Yang Y-L, Chuang J-H, et al. Inflammation-induced hepcidin is associated with the development of anemia and coronary artery lesions in kawasaki disease. J Clin Immunol 2012;32:746-752.

94. Pechlaner R, Kiechl S, Mayr M, et al. Correlates of serum hepcidin levels and its association with cardiovascular disease in an elderly general population. Clin Chem Lab Med 2016;54:151-161.

95. Galesloot TE, Holewijn S, Kiemeney LALM, et al. Serum hepcidin is associated with presence of plaque in postmenopausal women of a general population. Arterioscler Thromb Vasc Biol 2014;34:446-456.

96. Valenti L, Dongiovanni P, Motta BM, et al. Serum hepcidin and macrophage iron correlate with MCP-1 release and vascular damage in patients with metabolic syndrome alterations. Arterioscler Thromb Vasc Biol 2011;31:683-690.

97. Gustafsson $H$, Hallbeck $M$, Norell $M$, et al. Fe(III) distribution varies substantially within and between atherosclerotic plaques. Magn Reson Med 2014;71:885-892.

98. Li W, Xu L-H, Forssell C, et al. Overexpression of transferrin receptor and ferritin related to clinical symptoms and destabilization of human carotid plaques. Exp Biol Med 2008;233:818-826.

99. Mascitelli L, Goldstein MR. Hereditary hemochromatosis, iron, hepcidin, and coronary heart disease. Med Hypotheses 2014;82:402-403.

100. Sullivan JL. Do hemochromatosis mutations protect against ironmediated atherogenesis? Circ Cardiovasc Genet 2009;2:652-657.

101. Engberink MF, Povel CM, Durga J, et al. Hemochromatosis (HFE) genotype and atherosclerosis: increased susceptibility to iron-induced vascular damage in C282Y carriers? Atherosclerosis 2010;211:520-525.

102. Sasai $M$, Iso $Y$, Mizukami $T$, et al. Potential contribution of the hepcidin-macrophage axis to plaque vulnerability in acute myocardial infarction in human. Int J Cardiol 2017;227:114-121.

103. Saeed O, Otsuka F, Polavarapu R, et al. Pharmacological suppression of hepcidin increases macrophage cholesterol efflux and reduces foam cell formation and atherosclerosis. Arterioscler Thromb Vasc Biol 2012;32:299-307.

104. Finn AV, Nakano M, Polavarapu $R$, et al. Hemoglobin directs macrophage differentiation and prevents foam cell formation in human atherosclerotic plaques. J Am Coll Cardiol 2012;59:166-177.

105. Yao Y, Bennett BJ, Wang X, et al. Inhibition of bone morphogenetic proteins protects against atherosclerosis and vascular calcification. Circ Res 2010;107:485-494.

106. Kautz L, Gabayan V, Wang X, et al. Testing the iron hypothesis in a mouse model of atherosclerosis. Cell Rep 2013;5:1436-1442. 
107. Suzuki H, Toba K, Kato K, et al. Serum hepcidin-20 is elevated during the acute phase of myocardial infarction. Tohoku J Exp Med 2009;218: 93-98.

108. Park S-Y, Trinity JD, Gifford JR, et al. Mitochondrial function in heart failure: the impact of ischemic and non-ischemic etiology. Int J Cardiol 2016;220:711-717.

109. Basisty N, Dai D-F, Gagnidze A, et al. Mitochondrial-targeted catalase is good for the old mouse proteome, but not for the young: "reverse" antagonistic pleiotropy? Aging Cell 2016;15: 634-645.

110. Nicolas G, Viatte L, Lou D-Q, et al. Constitutive hepcidin expression prevents iron overload in a mouse model of hemochromatosis. Nat Genet 2003;34:97-101.
111. Preza GC, Ruchala $\mathrm{P}$, Pinon $\mathrm{R}$, et al. Minihepcidins are rationally designed small peptides that mimic hepcidin activity in mice and may be useful for the treatment of iron overload. J Clin Invest 2011;121:4880-4888.

112. Mleczko-Sanecka $K$, da Silva AR, Call D, et al. Imatinib and spironolactone suppress hepcidin expression. Haematologica 2017;102: 1173-1184.

113. Poli $M$, Asperti $M$, Naggi A, et al. Glycol-split nonanticoagulant heparins are inhibitors of hepcidin expression in vitro and in vivo. Blood 2014;123:1564-1573.

114. Li JJ, Meng X, Si HP, et al. Hepcidin destabilizes atherosclerotic plaque via overactivating macrophages after erythrophagocytosis. Arterioscler Thromb Vasc Biol 2012;32:1158-1166. 